\title{
A Knowledge Model for Automatic Configuration of Traffic Messages
}

\author{
Martin Molina ${ }^{1}$, Monica Robledo ${ }^{2}$ \\ ${ }^{1}$ Department of Artificial Intelligence, Technical University of Madrid \\ Campus de Montegancedo s/n, 28660 Boadilla del Monte (Madrid), Spain \\ mmolina@fi.upm.es \\ ${ }^{2}$ Artificial Intelligence Group, E.S.C.E.T, University Rey Juan Carlos \\ C/Tulipan s/n, 28993 Mostoles (Madrid), Spain \\ mrobledo@escet.urjc.es
}

\begin{abstract}
This paper describes a knowledge model for a configuration problem in the domain of traffic control. The goal of this model is to help traffic engineers in the dynamic selection of a set of messages to be presented to drivers on variable message signals. This selection is done in a real-time context using data recorded by traffic detectors on motorways. The system follows an advanced knowledge-based solution that implements two abstract problem solving methods according to a model-based approach recently proposed in the knowledge engineering field. Finally, the paper presents a discussion about the advantages and drawbacks found for this problem as a consequence of the applied knowledge modeling approach.
\end{abstract}

\section{Introduction}

In the past 15 years, researchers within the knowledge engineering field have paid special attention in knowledge share and reuse in order to decrease the effort in building large and complex knowledge based applications. One important conclusion of this research is that, to build a knowledge-based system, it is appropriate to follow a modeling approach to emulate how a human expert solves problems in a particular professional area. For this purpose, it is useful to describe such a model in a cognitive level (called knowledge level [1]) by using certain description entities closer to human thinking than computer processing.

These results carried out an interesting proposal about how to reuse problemsolving knowledge. Authors proposed the concept of problem solving method (PSM), an abstract cognitive structure specialized in classes of problems, derived from original proposals of different authors such a J. McDermott [2], B. Chandrasekaran [3] and J. Clancey [4]. Each PSM defines a knowledge intensive architecture together with the reasoning process to solve certain class of problems, such as parametric design, classification, diagnosis, etc. Since then, some initial PSMs were identified as standard typical intuitive reasoning processes followed by human experts in certain kind of problems [5]. For example, a diagnostic task can be solved by the cover-anddifferentiate PSM or a classification task can be solved by the establish-and-refine PSM. 
Thus, presently, there is a new generation of useful techniques in the knowledge engineering community to help in the development of complex and large knowledgebased systems. To mature and extend this new and emergent technology, it is required experimentation with real-world problems, whose results complement the dissemination of theoretical approaches and contribute to identify open issues that require further research. This paper contributes in this direction by presenting a real-world knowledge modeling experience in the domain of traffic control. The paper presents a knowledge model for automatic configuration of a set of traffic messages for a set of variable message signals, according to the current state of a road network.

The paper presents, first, the characteristics of the traffic problem. Then, the knowledge model is described, following a model-based approach to structure and organize the set of knowledge bases together with the strategies of inference. Then, the implementation for the city of Madrid is presented. Finally a discussion is included about the utility of the knowledge modeling approach for this problem.

\section{Real-time Traffic Management}

Control centers for traffic management are usually connected on-line to devices such as detectors on roads, cameras, traffic lights, etc., in such a way that operators can supervise the state of the road by consulting data bases with recent information from detectors and can modify the state of control devices. The effective use of these traffic monitoring and management facilities requires sophisticated support tools for online operators, to help them in dealing with the information complexity and the diversity of sensors and control devices. In the last decade, expert systems for decision support have been successfully introduced in this field [6], [7], [8], [9].

Figure 1 shows a typical infrastructure for real-time traffic control that can be found in different cities. There are detectors on major roads recording several traffic measures such as speed (Km/h), flow (vehicle/h or vehicle/min) and occupancy (percentage of time the sensor is occupied by a vehicle). The distance between successive sensors on a freeway is usually about 500 meters. The information arrives periodically to the control center (e.g., every minute). The control center receives also information about the current state of control devices.

Control devices include traffic signals at intersections, traffic signals at on-ramps, variable message signs (VMS) that can present different messages to drivers (e.g., warning about an existing congestion or alternative path recommendation), radio advisory systems to broadcast messages to drivers, and reversible lanes (i.e., freeway lanes whose direction can be selected according to the current and expected traffic demand). In the control center, operators interpret sensor data and detect the presence of problems and their possible causes. Problems are congested areas at certain locations caused by lack of capacity due to accidents, excess of demand (like rush hours), etc. In addition, operators determine control actions to solve or reduce the severity of existing problems. For instance, they can increase the duration of a phase (e.g., green time) at a traffic signal, or they may decide to show certain messages on some VMSs to divert traffic. 


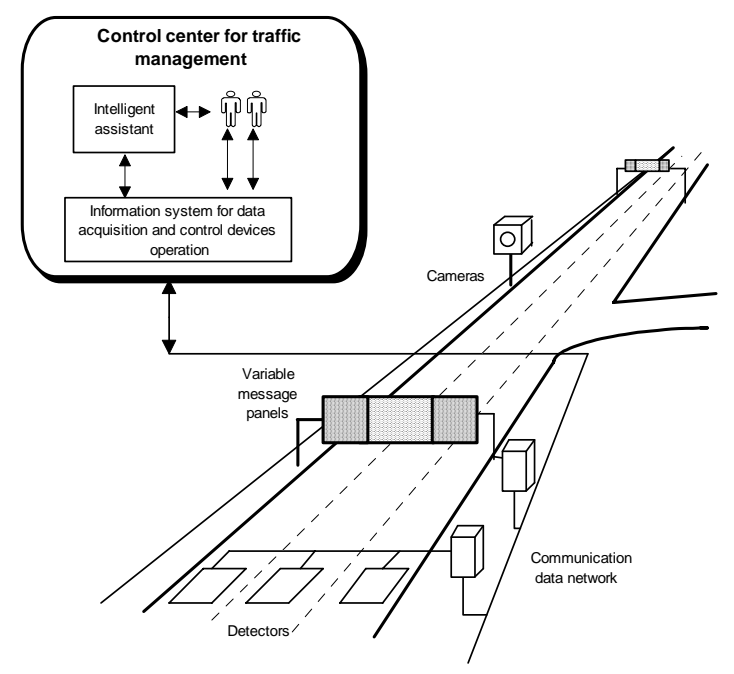

Fig. 1. Typical information infrastructure for real-time traffic management.

\section{A Knowledge Model for Message Configuration}

In order to help operators in this problem of traffic management, a computer system has been conceived as an intelligent assistant. The concept of intelligent assistant [10] emphasizes the fact that the role of the computer system is to help the user in decision-making but the final responsibility is taken by the user. To develop such a system, we followed a model-based approach in which the goal was to formulate a formal abstraction of the knowledge that we ascribe to operators for real-time traffic management. The operational version of this model is used to automatically reproduce by simulation part of the decision-making reasoning. For this purpose, we followed advanced knowledge modeling techniques (similar to the CommonKads approach [11]) that use a conceptual level for expertise description, with specific formal intuitive entities.

According to this, figure 2 shows a global view of the knowledge model that we designed for the traffic problem. This figure shows a hierarchy of tasks (circles) and methods (squares) with types of knowledge bases (at the bottom). The main task of the system is to recommend every moment an appropriate state of control devices. The top-level node corresponds to this global task called configure traffic messages. This is a configuration task where, from an initial situation (the traffic state at different locations), a complete design must be determined as a set of states for control devices (e.g., messages for each VMS panel). In this process, two general requirements have to be considered: (1) each traffic message must be consistent with the traffic state, and (2) each traffic message must be consistent with the rest of traffic messages. The qualitative nature of messages together with specific criteria (areadependent) based on a heuristic knowledge of the area for certain panels makes difficult the use of generic algorithmic solutions for this problem. 


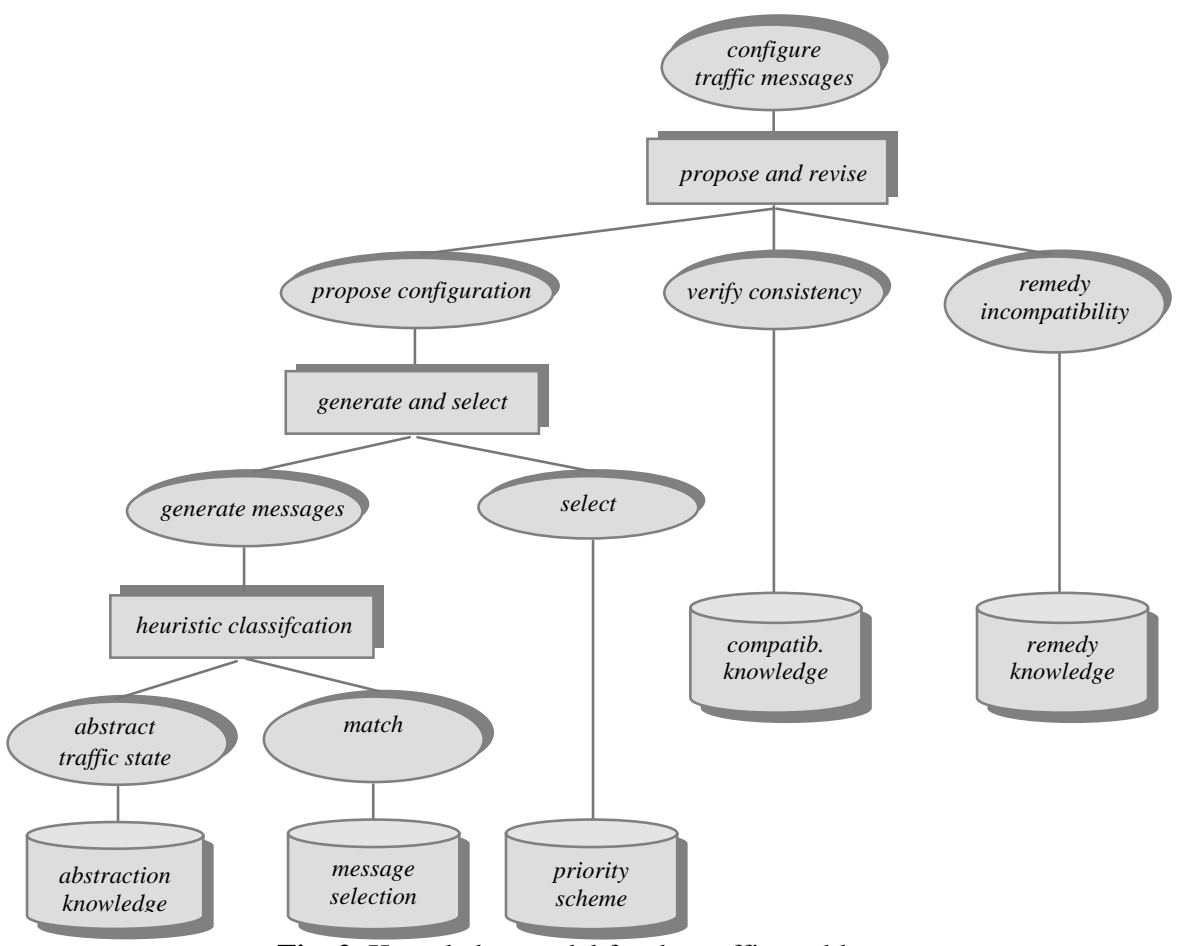

Fig. 2. Knowledge model for the traffic problem.

The usual way of reasoning in this problem follows a tentative search supported by specialized knowledge. Thus, in a first step, a set of messages is proposed based on the current state of the traffic. Then, the proposal is analyzed to check whether this proposal violates some constraints about coherence of messages (e.g., it is not acceptable to present too many messages about the same problem in consecutive panels). If so, a remedy is applied based on heuristic knowledge to modify the original proposal. This process is repeated until, if possible, a satisfactory configuration is found. This type of reasoning has been already identified and typified as a problem-solving method in the knowledge engineering literature and receives the name of proposeand-revise [12].

In particular, the abstract structure of this method assumes the existence of three kinds of knowledge: (1) derivation knowledge, that includes specific criteria to deduce a design from initial specifications, (2) compatibility knowledge, to verify a proposed design and includes a set of criteria to identify incompatible cases, and (3) remedy knowledge that includes a set of criteria about how to solve a violation detected in the design. According to this knowledge organization, the method decomposes the global configuration task into three sub-tasks: propose configuration, verify consistency and remedy incompatibility (see figure 2).

In its turn, the first sub-task is carried out by a specific method defined for this purpose that, first, generates proposals of messages (several messages for each panel) and, then, one of them is selected based on a priority scheme. To carry out the first step (generate messages) row data recorded by sensors need to be abstracted and 
related to candidate traffic messages. For this purpose, given the qualitative nature of the problem, the heuristic classification PSM [4] can be used. This method receives a set of observables (e.g., sensor data) and produces a set of solutions (e.g., proposals of traffic messages). The general version of this method includes three types of knowledge: (1) abstraction knowledge, to abstract the row data, (2) heuristic match knowledge, to classify abstracted data into classes of solutions and (3) refinement knowledge, to refine the classes of solutions into specific solutions. However, the particular version of the heuristic classification method for the traffic problem requires only two subtasks: abstract traffic state and match.

At the bottom level, figure 2 shows the set of knowledge types included in the model. There are a total of five specific types of knowledge bases that give support to the respective five subtasks. The general inference procedure makes use of the previous knowledge bases following three main steps:

1. Propose configuration. From the current traffic state, a set of potential messages is generated for each panel by using the abstraction knowledge and the message selection knowledge. For each panel, one message is selected by using the priority scheme.

2. Verify consistency. From the set of candidate messages and using the compatibility knowledge, the proposal is verified to check whether it satisfies the coherence criteria. If the proposal is coherent, the proposal is accepted as a final result. Otherwise, step 3 is performed.

3. Remedy incompatibility. From the detected incompatibilities, and using the remedy knowledge, a new set of candidate messages is proposed. For each incompatibility, the panel that must change the proposal is selected. Then, the loop starts again from step 1 selecting alternative messages and verifying the consistency.

This model can be considered as a global pattern that serves as a guide to acquire, organize and implement the specific knowledge of the system. The model divides the whole knowledge of the system in different categories (control knowledge, domain knowledge) according to the generic reused problem-solving strategies.

\subsection{Symbolic knowledge representation}

The previous model needs to be complemented with the specific symbolic representation used for each type of knowledge base. The availability of each knowledge base gives the required level of generality to apply this model for different traffic networks, by providing the specific control strategies for each case.

First of all, the model includes a conceptual vocabulary to establish the basic traffic terminology used by the knowledge bases. This vocabulary includes, among others, the following main concepts: (1) VMS panel $V_{i}$, to identify the set of VMS panels on the road, (2) message $M_{i}$, i.e., a particular message to be written on a particular panel, (3) road section $S_{i}$, that identifies a section of the road corresponding to a point where a detector is located, and (4) path $P_{i}$, that defines a path as sequence of road sections. 
The purpose of the first knowledge base, abstraction knowledge, is to deduce traffic characteristics of higher level of abstraction through data interpretation of information recorded by sensors. This knowledge base can be considered as logic implications to deduce values for attributes of sections or paths with a formulation like the following $(<C, A, V>$ means that the attribute $A$ of the concept $C$ takes the value $V$ ):

$<S_{i}$, Observable, $X_{i}>, \ldots,<S_{j}$, Observable, $X_{j}>, \ldots \rightarrow<S_{n}$, Abstraction, $X_{n}>$

$<S_{i}$, Abstraction, $X_{i}>, \ldots,<S_{j}$, Abstraction, $X_{j}>, \ldots \rightarrow<P_{k}$, Abstraction, $X_{k}>$

This format can include elaborated relations such as the following (for instance, to compute a particular travel time using a specific function $f$ ):

$<S_{i}$, speeed, $X_{i}>, \ldots,<S_{j}$, speed, $X_{j}>, \ldots, f\left(X_{i}, \ldots, X_{j}, \ldots\right)=X_{k} \rightarrow<P_{k}$, travel time, $X_{k}>$

The knowledge base for message selection is used to deduce one or several proposals of messages for each VMS panel according to the measures recorded by traffic detectors. This knowledge can be represented with the following type of sentences:

$<S_{i}$, Attribute $, X_{i}>, \ldots,<P_{j}$, Attribute $e_{j}, X_{j}>, \ldots \rightarrow<V_{k}$, message, $M_{k}>$

$<V_{i}$, message, $M_{i}>, \ldots,<V_{j}$, message, $M_{j}>, \ldots \rightarrow<V_{k}$, message, $M_{k}>$

The previous knowledge relates the traffic state and the signal state and it must be formulated with the important requirement that the relations do not have to include loops to avoid circular calculation, according to the propose and revise strategy. This symbolic representation provides the required flexibility to write specific cases corresponding to each type of message (congestion, incident, travel time, etc.). Together with this, it is necessary to have criteria to select one message within a set of candidate messages. For this purpose the priority scheme, is defined as follows:

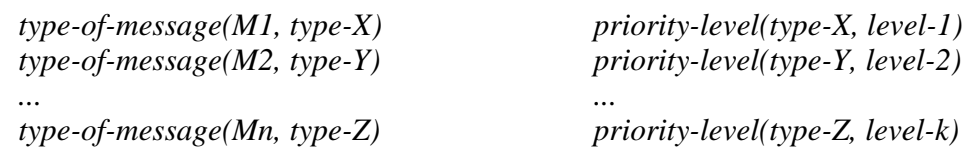

... priority-level(type-Z, level-k)

For instance the most prioritary level can be assigned for the messages of the type incident, the second level to the traffic jam warning type, third level for destination travel time, and so on.

The compatibility knowledge base includes conditions that establish unacceptable combinations of messages in order to avoid inconsistency between messages. This knowledge is formulated with constraints as following:

constraint $_{k}$ : $<V_{i}$, message, $M_{i}>, \ldots,<V_{j}$, message, $M_{j}>, \ldots,<$ traffic conditions $>$

The constraint includes a set of messages for panels together with a logic expression (that optionally can include values about the traffic state) to define an incompatible message combination. This representation is flexible enough to include both general and particular conditions about compatibility according to the requirements of each traffic network. For instance, it is useful to formulate that it is not acceptable to present the same message on two specific panels.

Finally, the remedy knowledge base includes the criteria with which the detected inconsistencies are solved by modifying a proposed configuration. In this case, this means to select which panel is required to change the message in order to avoid the inconsistency. This is represented by:

constraint $_{k},<$ traffic conditions $>\rightarrow$ panel $V_{j}$ 
which means that, to solve the violation represented by constraint $t_{k}$ panel $V_{j}$ should change its message (when certain traffic conditions are present).

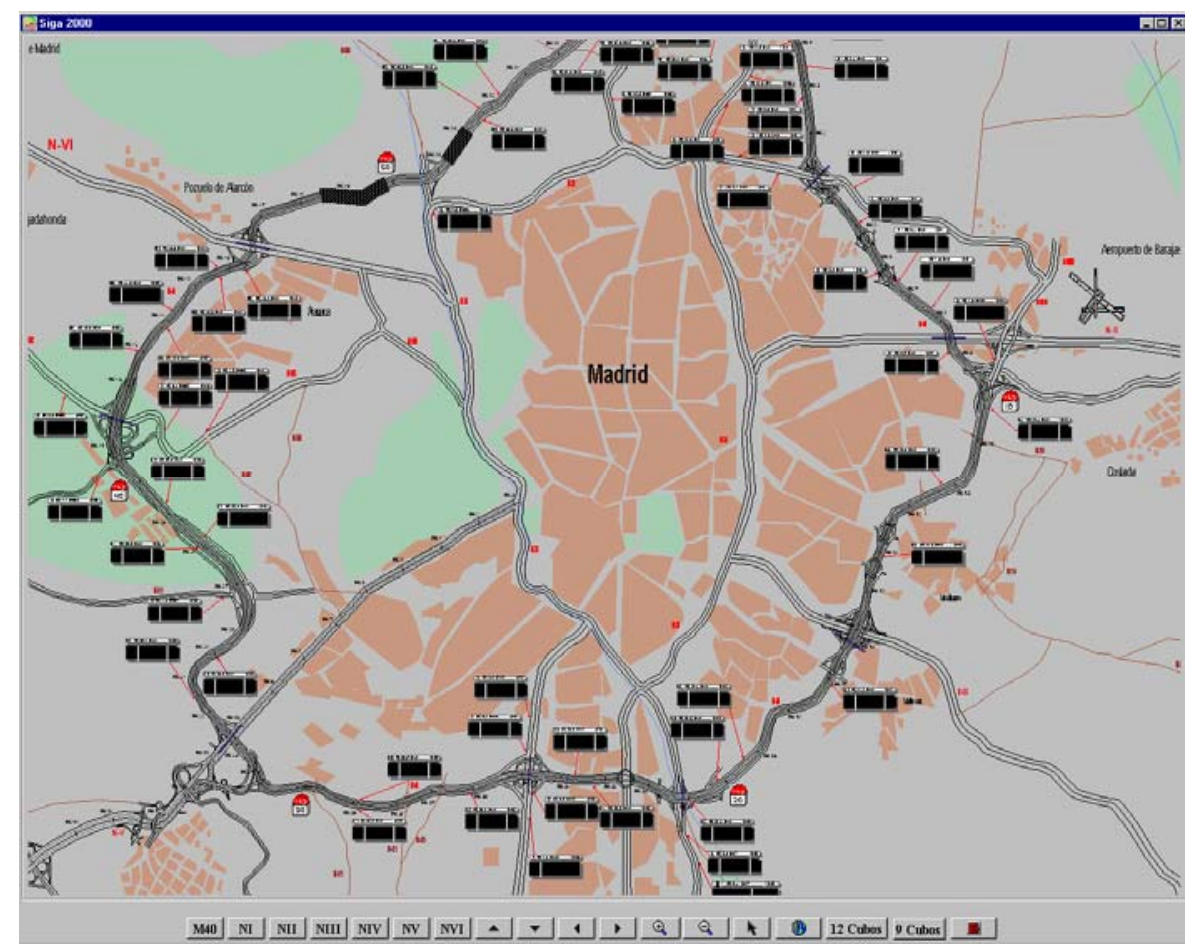

Fig. 3:The M-40 motorway of Madrid with variable message panels

\section{Experience and example}

Following this general model, a system was developed for the city of Madrid, to control part of the M-40 Urban Ring (figure 3). This road is a motorway of around 60 $\mathrm{Km}$ in both ways that includes a total of 63 panels to present messages to drivers. The road includes the typical information infrastructure for real-time traffic management and operators must decide on real-time the set of messages to be presented in those panels according to the behavior of the road (traffic jams, incidents, etc.).

Figure 4 presents an example of the output produced by the system. It corresponds to an example where the system receives input data like the following:

< detector D41, speed, 45>

$<$ detector D41, occupancy, 25>

$<$ detector D41, flow, 38>

$<$ detector D44, speed, 25>

$<$ detector D44, occupancy, 40>

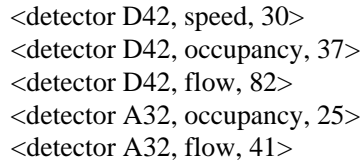

According to this input data, a problem is detected between road detectors D41 and D44 given that a decrease of the speed is observed, and due to that the travel time to 
the road M-607 is a high travel time compared with a normal state of the traffic. The high measure of the detector A32 (corresponding to an off-ramp) is used to deduce that there is a traffic jam at $\mathrm{N}-\mathrm{V}$ Exit.

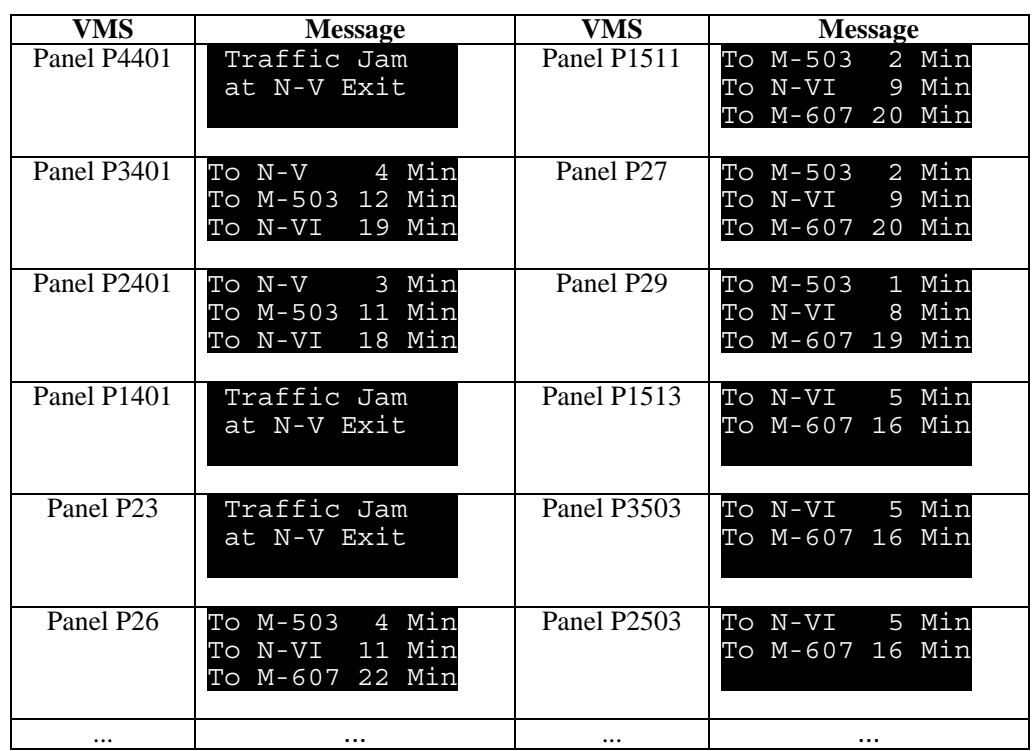

Fig. 4: Example of outputs produced by the system (English version)

This system was implemented by using a knowledge modeling software tool, called KSM (Knowledge Structure Manager) developed by our own group [13] and it was integrated with the rest of the information system to run on-line at the Traffic Control Center of the city of Madrid. The specific software was designed following an object oriented design, and was implemented in $\mathrm{C}++$. There were about 3000 lines of code $\mathrm{C}++$ specific for the system and about 70000 lines of reused code $\mathrm{C}++$, corresponding to the KSM tool for knowledge representation.

\section{Discussion and conclusions}

In summary, the qualitative nature of traffic messages together with specific criteria area-dependent, corresponding to heuristic knowledge of the area for specific traffic panels, makes difficult a solution based on general algorithms. Thus, an approach from the field knowledge-based systems, in particular problem-solving methods for configuration design problems, has been useful to solve this problem.

To analyze the problem, it was very useful to reuse generic problem-solving methods according to a model-based knowledge acquisition process. However, for design and implementation it was necessary to provide additional solutions. This was performed by using our own knowledge-modeling tool, KSM, that incorporates some of the recent advances about knowledge modeling and provides reusable software components. 
As a difficulty, the model-based approach for knowledge engineering requires to revise and know a set of potential problem-solving methods that are defined using a particular level of abstraction. To understand totally such a descriptions, it is required additional work using diverse information sources. To decrease this, it is still necessary to make an effort in the knowledge engineering community to bring together and standardize all this technology as a continuation of initiatives such as [Breuker, Van de Velde, 94].

The proposed model is open. It allows developers to define particular control strategies for traffic message configuration, thanks to the availability of a set of knowledge bases with a declarative representation. At the same time, instead of having a unique global knowledge base, the knowledge is distributed in different specialized bases, according to intuitive problem-solving methods, that makes easier the total comprehension of the systems and contributes to keep the consistency.

On the other side, the applied method presents some similarities with the idea of Truth Maintenance Systems TMS. In general, a TMS ([14], [15]) keeps a predefined set of declarations in terms of consistency, i.e. if in a given state the set of assumed TMS nodes is inconsistent the TMS identifies the assumed nodes responsible for the inconsistency and modifies the assumptions to keep the compatibility. However, the specific model presented here is a solution that is defined at a lower level of abstraction closer to the design problem, which make easier its application, and it proposes an explicit representation for heuristic knowledge to remedy violations, which can make easier its application for the traffic control problem.

Finally, the model presented in this paper can be considered an innovative development within the field of transport that takes advantage of the recent advanced proposals in the domain of knowledge engineering. The solution makes available on real time strategic knowledge to help operators in selecting the most appropriate state of control devices, taking care every moment the compatibility between the road state and the signal state. This development was carried out for the M-40 urban ring for the city of Madrid and it was installed on-line at the Traffic Control Center of the city.

\section{Acknowledgments}

The authors would like to thank the Dirección General de Tráfico of Spain who provided financial support for the development of this system. The INDRA company provided technical support for real-time operation in the traffic control center of Madrid.

\section{References}

1. Newell A.: "The Knowledge Level" In Artificial Intelligence Vol 18 pp. 87-127, 1982.

2. McDermott J.: "Preliminary Steps Toward a Taxonomy of Problem Solving Methods". In "Automating Knowledge Acquisition for Expert Systems", S.Marcus ed., Kluwer Academic, Boston, 1988.

3. Chandrasekaran B., Johnson T.R, Smith J.W.: "Task Structure Analysis for Knowledge Modeling", Communications of the ACM, 35 (9), 124-137. 1992.

4. Clancey W.: "Heuristic Classification”. Artificial Intelligence 27, 1985.

5. Breuker J., Van de Velde W.: "CommonKADS Library for Expertise Modelling: Reusable Problem Solving Components". IOS Press. 1994. 
6. Cuena J., Ambrosino G., Boero M.: “A general Knowledge-based architecture for traffic control: the KITS approach”. Proceeding of the International Conference Artificial Intelligence in Transportation Engineering. San Buenaventura, California.

7. Deeter D.L., Ritchie S.G.: "A prototype real-time expert system for surface street traffic management and control”. ASCE, Third International Conference on Applications of Advanced Technologies in Transportation Engineering. Seattle, Washington, USA, 1993.

8. Molina M., Logi F., Ritchie S., Cuena J. : "An architecture integrating symbolic and connectionist models for traffic management decision support”. Proceedings of the VI International Conference on Applications of Advanced Technologies in Transportation Engineering.

9. Molina M., Hernández J., Cuena J.: “A Structure of Problem-solving Methods for Real-time Decision Support in Traffic Control”. Interational Journal of Human and Computer Studies (Academic Press) N.49, 577-600, 1998.

10. Boy, G., Gruber T.R.: "Intelligent Assistant Systems: Support for Integrated HumanMachine Systems” Technical Report KSL 90-61, Knowledge Systems Laboratory, Computer Science Department, Stanford University, 1990. Also in the proceedings of 1990 AAAI Spring Symposium on Knowledge-Based Human-Computer Communication, March 1990, Stanford University.

11. Schreiber G., Akkermans H., Anjewierden A., de Hoog R., Shadbolt N., Van de Velde W., Wielinga B.: "Knowledge Engineering and Management. The CommonKADS Methodology” MIT Press, 2000.

12. Marcus S., McDermott J.: "SALT: A Knowledge Acquisition Language for Propose-andRevise Systems”. Artificial Intelligence, Vol 39, No.1, 1989.

13. Cuena J., Molina M.: "The Role of Knowledge Modeling Techniques in Software Development: A General Approach based on a Knowledge Management Tool”. International Journal of Human and Computer Studies. No.52. pp 385-421. Academic Press, 2000.

14. Mc. Allester: "An Outlook to Truth Maintenance" AI Memo 551 MIT Cambridge Mass, 1980.

15. Doyle: "A Truth Maintenance System" Artificial Intelligence 12. Elsevier Science Publishers B.V. (North Holland) 1979. 